\title{
Analysis on the Fluid Flow in Vortex Tube with Vortex Periodical Oscillation Characteristics
}

\author{
Bo Zhang*, Xiangji Guo, Zhuqiang Yang \\ (School of Energy and Power, Dalian University of Technology, Liaoning, China) \\ *Corresponding Author: Dr. Bo Zhang, Email: zhangbo@dlut.edu.cn, Tel/Fax: +86 411 \\ 84777601)
}

\begin{abstract}
To reveal the energy transferring mechanism in the vortex tube, which is an interesting phenomenon in the area of heat and mass transfer, numerical simulation and analysis of the dynamic fluid flow were employed. In contrast to the previous static study, the focus of this paper is the dynamic process, or the oscillation, of the secondary circulation layer. Based on the fluid flow results derived from the unsteady 3-D computation, the existence of the forced or Rankine vortex was confirmed, which also verified the certainty of reverse flow in the cold end of the vortex tube. Then, the oscillation of the boundary layer of the central recirculation zone was emphasized and the periodical vibrating of the fluid flow within the secondary circulation zone, varying of its boundary, and the typical frequencies of points on a cross section were provided. Based on these results, a novel energy transferring mechanism in the vortex tube was proposed, under the condition that stable oscillation of the boundary layer is the dominant mechanism for the heat and mass transfer process.
\end{abstract}

Keywords: heat transfer; energy separation; oscillation; vortex tube; vortex breakdown 


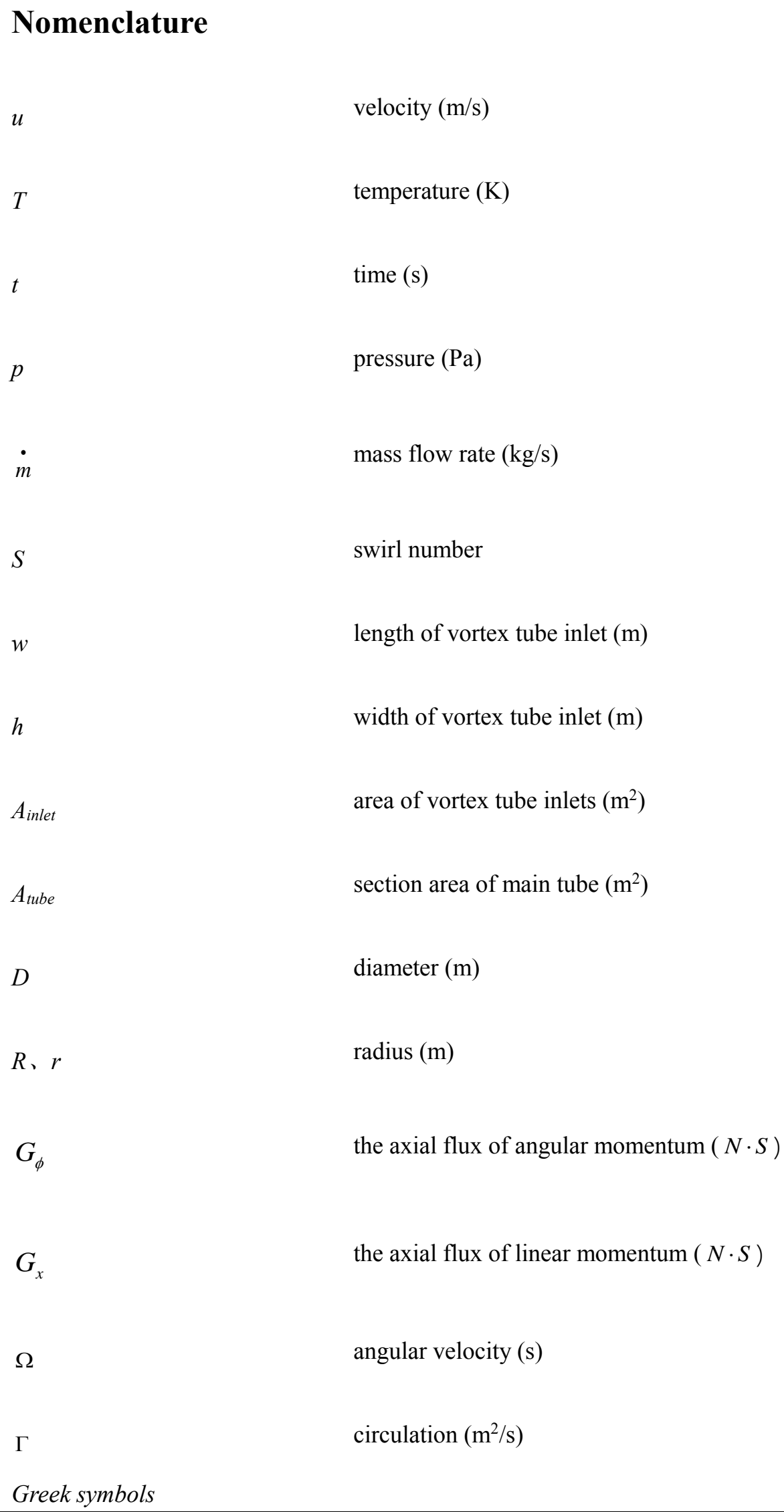




\begin{tabular}{|ll|}
\hline$\xi$ & cold mass fraction \\
$\tau$ & density $\left(\mathrm{kg} / \mathrm{m}^{3}\right)$ \\
$\varphi$ & time (s) \\
$\omega$ & phase angle (rad) \\
Subscripts & Vorticity $\left(\mathrm{s}^{-1}\right)$ \\
$\mathrm{c}$ & cold gas \\
$\mathrm{h}$ & hot gas \\
$\mathrm{t}$ & tangential direction \\
$\mathrm{i}$ & main tube \\
$\mathrm{x}, \mathrm{Z}$ & axial direction \\
&
\end{tabular}

\section{Introduction}

Though the configuration of a vortex tube is simple, it exhibits a unique phenomenon of energy (or temperature) separation with a single source of pressurized gas to instantly generate cold and hot streams simultaneously. Figure 1 shows the structure of a counter-flow vortex tube, which contains a main tube with tangential injection in the vortex chamber, a cold exit near the inlet, and a hot exit far from the inlet containing a control valve to change the flowrate proportion between both exits and achieve different effects of energy separation. The instant cooling or heating effects are produced without any moving parts or chemical reactions inside the vortex tube, which lowers the amount of maintenance necessary and increases the service life. Due 
to the high swirl velocity caused by the tangential injection, gas separation and undesired condensable component removal from a gas stream have become areas of research interest. The cooling effects have made vortex tube advantageous for use in liquefaction of natural gas.

Since the vortex tube was invented by Georges J. Ranque [1] in the 1930s, the quantitative analysis and mathematical model of energy separation have not been fully developed due to the intense turbulent swirling flow in the vortex tube. A better description of the flow structure and more reasonable explanation of the energy separation have limited broader use and higher thermal efficiency of the vortex tube. Studies of the vortex tube have been chronologically reviewed by Gustol [2], Eiamsaard [3], Xue [4] and Thakare [5] in recent decades. Among the different explanations or hypothesis models, the secondary circulation, resulting from part of the cold air that is forced back by the plug must return to the hot end, has been experimentally and numerically confirmed. It has been suggested that the thermal energy transferred from the inner flow to the outer flow is a refrigeration cycle or heat pump, as proposed by Ahlborn $[6,7]$. Xue [8-10] also concurred with the presence of secondary circulation and attributed the energy separation to partial stagnation.

Although there have been intensive studies on fluid flow and heat transfer within the vortex tube, a clear explanation of the process in which heat is transferred from the low-temperature region in the center to the outer higher-temperature region has not been provided. Most of the previous research is based on static gradient analysis, such as pressure, temperature or velocity.

Other interesting investigations analyzing fluid flow and energy transfer within the vortex tube lead to another research direction. Acoustic streaming in the core-flow was studied by Kurosaka [11], who believed the streaming induced by orderly disturbances in the swirl flow deforms the Rankine vortex into a forced vortex, which leads to energy separation in the radial direction. Liew $[12,13]$ established a model similar to Maxwell's demon with adiabatic compression and expansion caused by turbulent eddies. In Liew's model, the gas pocket was moved back-and-forth between the wall and axis to exchange energy with the flow in the core and periphery, resulting 
in the temperature separation. From their work, evidence of swirl dynamics and analysis on the vibration of swirl flow was introduced.

In this paper, the fluid flow is analyzed with the knowledge of swirl flow. From simulation and calculation, it is clear that reverse flow within the vortex tube is a natural feature of the strong swirl flow. The vortex breakdown and leakage of partial pressurized gas to the cold exit is not taken into consideration. In addition, the important inherent characteristic of vortex breakdown cannot be considered time-stable, and the precessing vortex core $(P V C)$ phenomenon is studied. There was some evidence of $P V C$ contributing to the energy separation in Kurosaka's experiment[11], where acoustic suppressors were used to weaken the $P V C$ and resulted in distinct deterioration of the temperature separation effect. Based on these ideas, a new energy transfer model within the vortex tube is presented in this paper.

In addition to providing analysis on the energy separation phenomenon of the vortex tube, we theorize that the combination of pulsation flow structure and energy transfer may contribute to the common studies of fluid flow and heat transfer.

\section{Numerical model and verification}

To analyze the swirl flow and its dynamic features, the unsteady computation fluid dynamic method was implemented to acquire fluid field data.

\subsection{Geometry Model}

In this paper, the vortex tube used by Dincer et al. [14] in experimental studies and by Baghdad et al.[15, 16] in steady-state numerical investigations was used to make comparisons and verification. The geometry of the vortex tube is shown in Figure 1. 


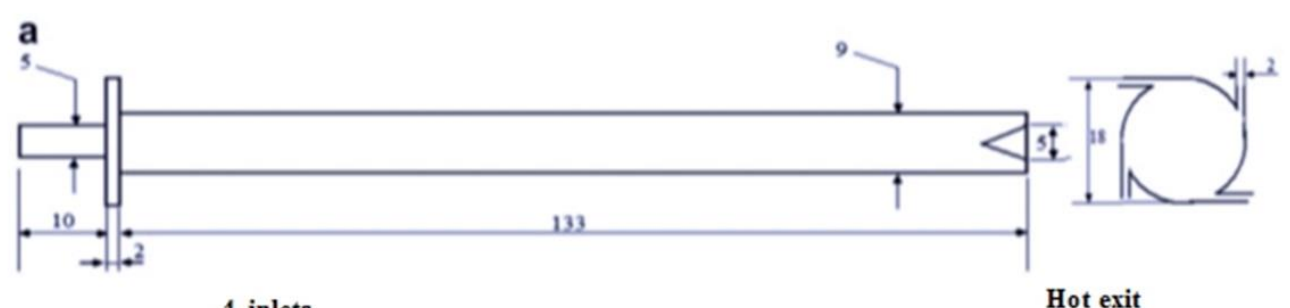

b

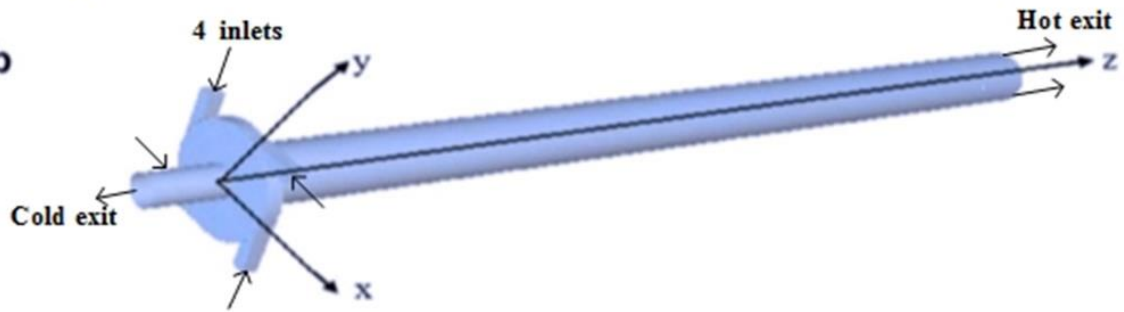

Figure 1 Geometry model: a. schematic representation and dimensions (in mm); b. axis system from Baghdad et al.

\subsection{Governing Equations of numerical model to the fluid flow}

The mass, momentum, energy conservation and state equation solved for compressible turbulent flows in the vortex tube are as follows:

$$
\begin{gathered}
\frac{\partial \rho}{\partial t}+\frac{\partial}{\partial x_{i}}\left(\rho \overline{u_{i}}\right)=0 \\
\frac{\partial}{\partial t}\left(\rho \overline{u_{i}}\right)+\frac{\partial}{\partial x_{i}}\left(\rho \overline{u_{i} u_{j}}\right)=\frac{\partial}{\partial x_{j}}\left(\mu \frac{\partial \overline{u_{i}}}{\partial x_{j}}-\rho \overline{u_{i}^{\prime} u_{j}^{\prime}}\right)-\frac{\partial p}{\partial x_{i}} \\
\frac{\partial}{\partial t}(\rho H)+\frac{\partial}{\partial x_{i}}\left(\rho \overline{u_{j}} H\right)=\frac{\partial p}{\partial t}+\frac{\partial}{\partial x_{j}}\left(\frac{k}{c_{p}} \frac{\partial H}{\partial x_{j}}-\rho \overline{u_{i}} H\right)
\end{gathered}
$$

$$
p=\rho R T
$$

where $\rho$ is the fluid density, $u$ is the fluid velocity, $p$ is the static pressure, $H$ is the total enthalpy, $k$ and $c_{p}$ are the thermal conductivity and specific heat of the fluid, respectively, $R$ is the ideal gas constant, and $T$ is the gas temperature.

The transition Reynolds Stress Model was used to simulate the turbulence in the following analysis. Feasibility and validity of this turbulent model in simulating swirl flow can be found in related publications and discussion [17-20]. 


$$
\begin{aligned}
& \frac{\partial}{\partial t}\left(\rho \overline{u_{i}^{\prime} u_{j}^{\prime}}\right)+\frac{\partial}{\partial x_{k}}\left(p u_{k} \overline{u_{i}^{\prime} u_{j}^{\prime}}\right)=-\left[\rho \overline{u_{i}^{\prime} u_{j}^{\prime} u_{k}^{\prime}}+\overline{p^{\prime}\left(\sigma_{k j} u_{i}^{\prime}+\sigma_{i k} u_{j}^{\prime}\right)}\right] \\
& -\rho\left(\overline{u_{i}^{\prime} u_{j}^{\prime}} \frac{\partial u_{j}}{\partial x_{k}}+\overline{u_{j}^{\prime} u_{k}^{\prime}} \frac{\partial u_{i}}{\partial x_{k}}\right)+\overline{p^{\prime}\left(\frac{\partial u_{i}^{\prime}}{\partial x_{j}}+\frac{\partial u_{j}^{\prime}}{\partial x_{i}}\right)}-2 \mu \overline{\frac{\partial u_{i}^{\prime}}{\partial x_{k}} \frac{\partial u_{j}^{\prime}}{\partial x_{k}}}
\end{aligned}
$$

$D_{T, i j}$ can be modeled by the generalized gradient-diffusion model of Daly and

Harlow [21],

$$
D_{\tau, i j}=c_{s} \frac{\partial}{\partial x_{k}}\left(\rho \frac{k \overline{u_{k}^{\prime} u_{j}^{\prime}}}{\varepsilon} \frac{\partial \overline{u_{i}^{\prime} u_{j}^{\prime}}}{\partial x_{j}}\right)
$$

The linear model of Launder [22] was applied to model the pressure-strain term, $\phi_{i j}$, and the classical approach of modeling $\phi_{i j}$ can be calculated as:

$$
\phi_{i j}=\phi_{i j, 1}+\phi_{i j, 2}+\phi_{i j, w}
$$

where, $\phi_{i j, 1}$ is the slow pressure-strain term, also known as the return-to-isotropy term, $\phi_{i j, 2}$ is the rapid pressure-strain term, and $\phi_{i j, w}$ is the wall-reflection term.

The wall treatment of Reynolds Stress Model was based on the standard wall functions from the work of Launder and Spalding [23], which has been widely used in industrial flows.

\subsection{Boundary Conditions}

The motive inlet pressure was set as $260.0 \mathrm{kPa}$, derived from one case of Dincer's experiment, and the pressure of the cool discharged air was set to atmospheric pressure. To obtain different flow ratios between the cold and hot discharge, the boundary condition of the hot discharge pressure was variously set between $13.0 \mathrm{KPa}$ to $85.0 \mathrm{KPa}$ to simulate the opening of the control valve of the vortex tube. Thus, the desired flow ratio was derived by attempting different hot discharge pressures. The mass flux ratio between the cold stream and the total inlet mass flux $\xi$ is defined as,

$$
\xi=\frac{\dot{m}_{e}}{\dot{m}_{i}}
$$

The motive stream was compressible air at $300.0 \mathrm{~K}$ with a turbulent intensity of $5 \%$. All of the walls were adiabatic.

\subsection{Grid Generation and Computational Method}



as shown in Figure 2.

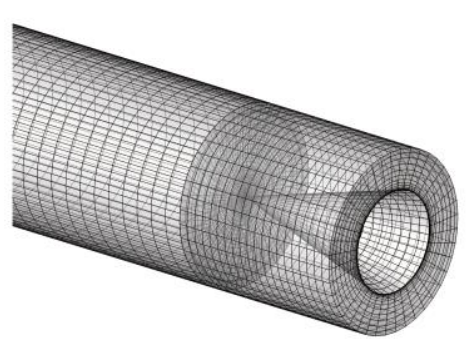

Figure 2 Computational mesh of the vortex tube.

A pressure based, second-up wind discretized method was adopted to solve the energy, momentum and turbulence equations. The PISO method was used to solve for the pressure and velocity coupling. The steady-state flow field was originally computed after relative errors below $10^{-5}$, and then the computation continued using an unsteady solver with a time step of $10^{-5} \mathrm{~s}$ to detect frequencies in the $\mathrm{kHz}$ range. To speed up the computation procedure, Non-Iterative Time Advancement (NITA) technology was used in the iteration process, where the max iteration steps were not required but automatically adjusted during every time step to confirm convergence.

\subsection{Validation of the Numerical Model}

The time-averaged total temperature difference was considered the crucial parameter for the grid-independence study. In the unsteady computation, the total temperature difference between the cold stream and hot stream discharged from the vortex tube was defined as, $\Delta T_{\text {total }, h c}=\bar{T}_{\text {tatal }, h}-\bar{T}_{\text {tatal }, c}[24]$, where was the time averaged on the exit areas defined as $\bar{T}_{\text {total }}=\frac{1}{\tau} \int_{t=t_{0}}^{t=t_{0}+\tau} T_{\text {total }} d t$ (Where $t_{0}$ is any time when the transient calculation reaches a quasi-steady state condition, i.e., the limit cycle oscillation). The time averaging was performed over 5,000 time-steps, $10^{-5} \mathrm{~s}$ per time-step, for a total of $0.05 \mathrm{~s}$. Calculations on the motive pressure of $260.0 \mathrm{kPa}$ were carried out, and the results were compared with the experimental data of Dincer et al.[14] and with steady RSM simulation data of Baghdad et al. [16]. The total temperature differences derived from numerical predictions and experimental data are compared in Figure 3. The 
experimental results increase linearly while the numerical results increase and then decrease at larger mass fractions. The results for cold mass fractions between 0.6-0.8 were not available from experimental data, but the main trend of $\bar{T}_{\text {tatal }}$ with $\xi$ was similar and relative errors were within $5 \%$ of experimental results within the cold mass fraction region from 0.15 to 0.55 , and the unsteady $T-R S M$ presented better than steady $R S M$. The flow field in the vortex tube became unstable at very small or large cold mass fractions and could not achieve the typical energy separation. Thus, most numerical simulations and theoretical analyzations would predict ambiguous results at these operating conditions [13]. In this paper, T-RSM outperformed RMS.

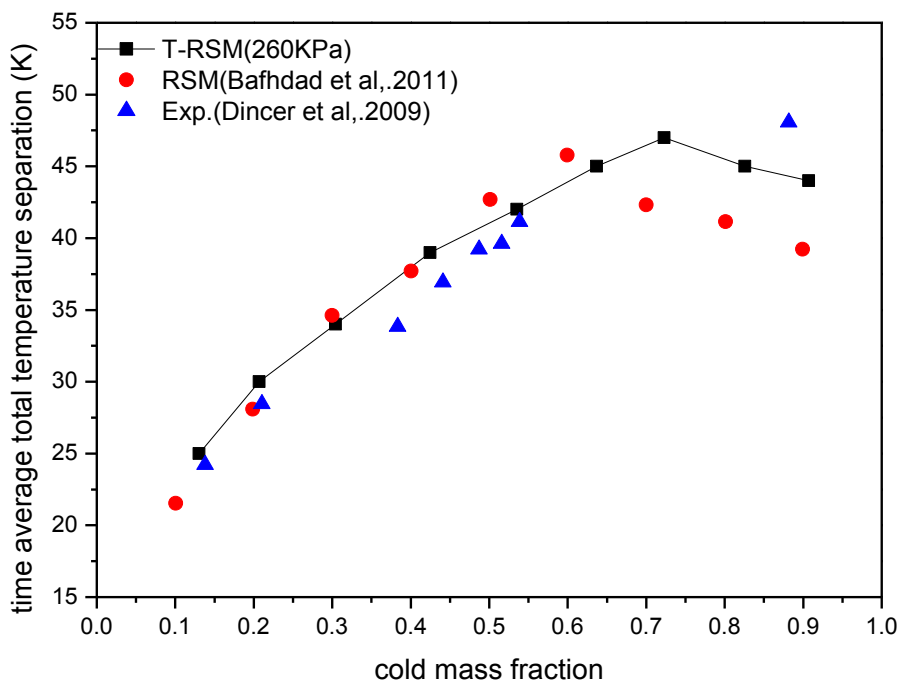

Figure 3 Performance of T-RSM in predicting experimental measurements and comparing with RSM

To verify the independence of the grid system, for a case of $P_{i n}=260.0 \mathrm{kPa}$ and $\xi$ $=0.3\left(P_{\text {hot } \_ \text {out }}=33 \mathrm{kPa}\right)$, the grids were refined using self-adapted technology according to the velocity gradient in Fluent 15.0. Simulations were carried out for four different grid sizes: 123,269 nodes, 176,621 nodes, 252,560 nodes, 430,252 nodes. Increasing the number of nodes beyond 252,560 does not significantly increase accuracy (Figure 4). In this paper, all results are with a grid size of 252,560 nodes. 


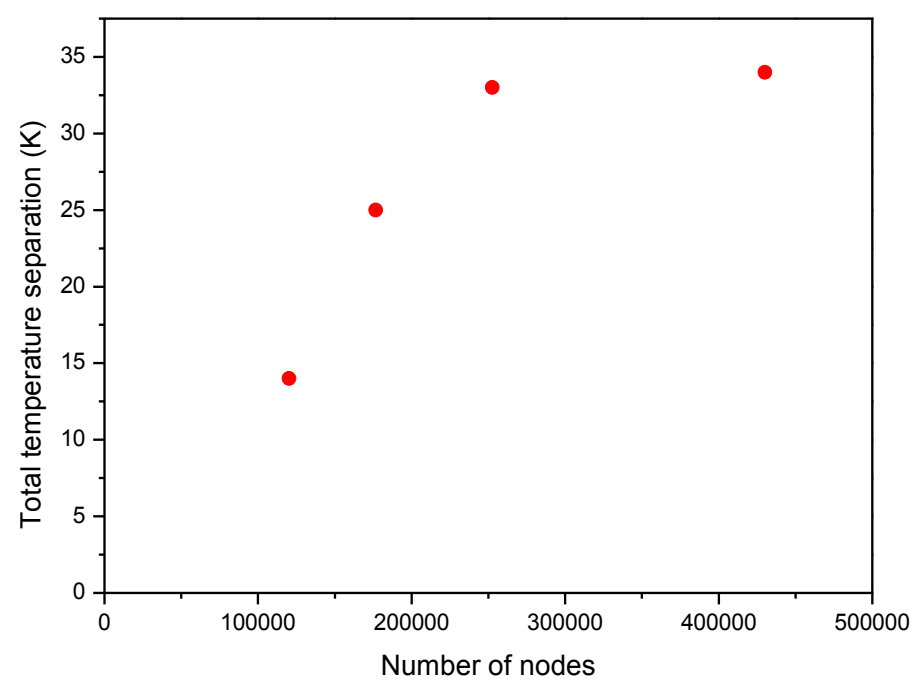

Figure 4 Grid size dependence study on time average total temperature separation (case for $P_{\text {in }}=260.0 \mathrm{kPa}, \xi=0.3$, time average was performed for 5,000 time steps)

\subsection{Discussion on the difference between steady state and unsteady state results}

The instantaneous axis velocity, $u_{z}$, contour on the meridional plane when the dynamic convergence was reached is shown in Figure 5. The contours show that axial symmetry did not completely exist and the unsteady character is distinguishable. However, the time-average velocity field is also important in determining the basic vortex structure in the vortex tube. The contour of the time-averaged velocity over $0.05 \mathrm{~s}$ is shown in Figure 6, in which axial symmetry is well presented.

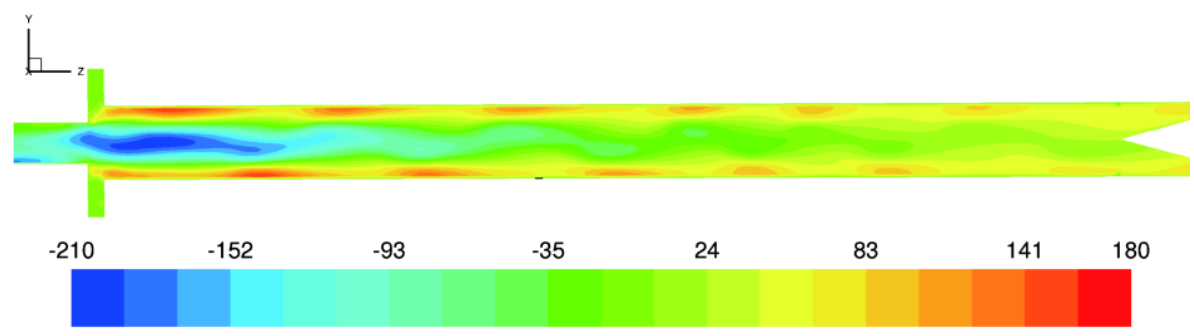

Figure 5 Instantaneous axial velocity contours in the meridional plane (case for $P_{\text {in }}=260 \mathrm{kPa}, \xi=0.3$.) 


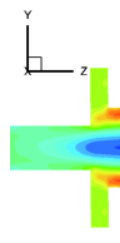

$-200$ $-98$ $-47$

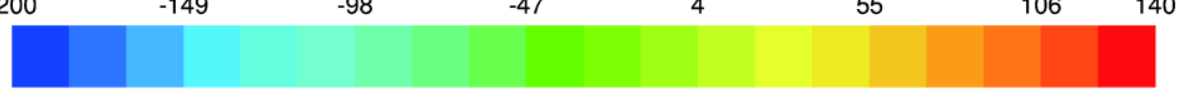

Figure 6 Time averaged axial velocity contours in the meridional plane (case for $P_{\text {in }}=260 \mathrm{kPa}, \xi=0.3$. Time average was covered for $0.05 \mathrm{~s}$.)

For meridional sectional planes with Z-axis locations of 1D, 4D, 7D and 11D, the axial velocity distribution on radii, from -0.0045 to $0.0045 \mathrm{~m}$, are shown in Figure 7 . The time-averaged axial velocity was defined as $\bar{u}_{z}=\frac{1}{\tau} \int_{t=t_{0}}^{t=t_{0}+\tau} u_{z} d t$. For these three sectional planes under the conditions, $P_{i n}=260 \mathrm{kPa}$ and $\xi=0.3$, the maximum timeaveraged axial velocities were $136.8 \mathrm{~m} / \mathrm{s}, 112.3 \mathrm{~m} / \mathrm{s}, 81.2 \mathrm{~m} / \mathrm{s}$ and $65.6 \mathrm{~m} / \mathrm{s}$ downstream near the wall. Near the centerline, the time-averaged axial velocities were in the opposite Z-axis direction and reached maximum absolute magnitudes of $-198.3 \mathrm{~m} / \mathrm{s}$, $82.4 \mathrm{~m} / \mathrm{s},-29.9 \mathrm{~m} / \mathrm{s}$ and $8.9 \mathrm{~m} / \mathrm{s}$, in which the negative sign denotes the opposite Z-axis direction. The reverse flow accelerated at the cold end due to the adverse pressure gradient. The vortex breakdown phenomenon was confirmed by the presence of the reverse flow.

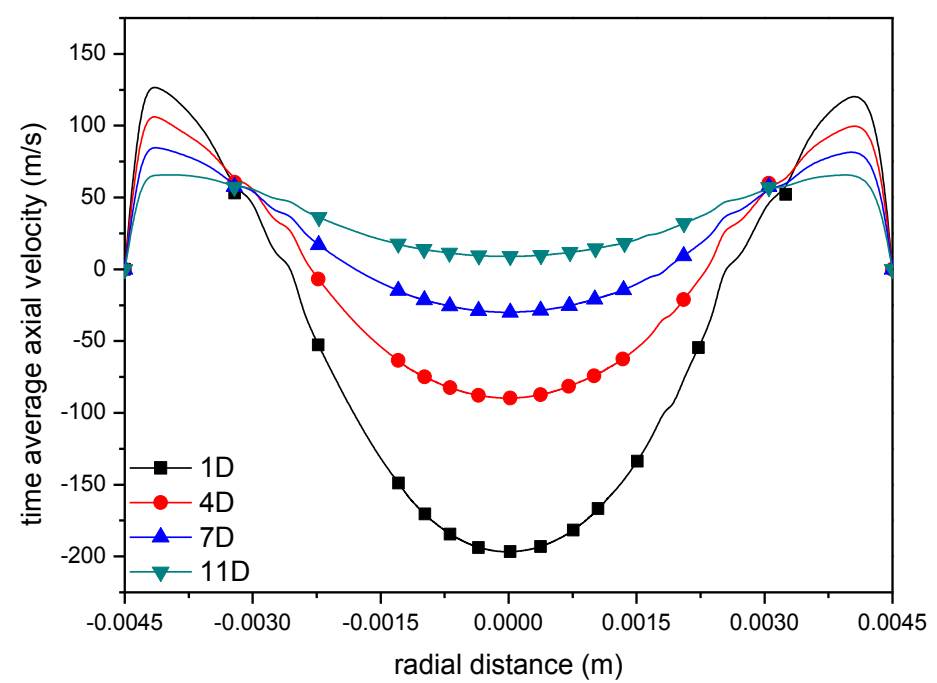
(case for $P_{\text {in }}=260 \mathrm{kPa}, \xi=0.3$. Time average was covered for $0.05 \mathrm{~s}$ ) 


\section{Analysis of vortex breakdown in the vortex tube}

202

203

204

205

206

207

208

209

\subsection{Vortex breakdown in the vortex tube}

The swirl flow process in a vortex tube, which had been described by Gupta [25] and Gustol [2], was clearly regenerated. To investigate swirl flow characteristics, the tangential velocity, $u_{t}=\Omega r$ ( $\Omega$ denotes the angular velocity, $r$ is the radius $)$, was used to distinguish a forced vortex from a free vortex, which often referred to the tangential velocity gradient. The forced vortex formed in the center of the vortex tube while the free vortex formed in the outer region of the vortex tube. A Rankine vortex, or combined vortex, is described as $u_{t}=\frac{C}{r}\left[1-\exp \left(-\frac{r^{2}}{r_{0}^{2}}\right)\right]$ (where the constants $C$ and $r_{0}$ characterize the vortex intensity and radial coordinate) [26] and is shown in Figure 8.

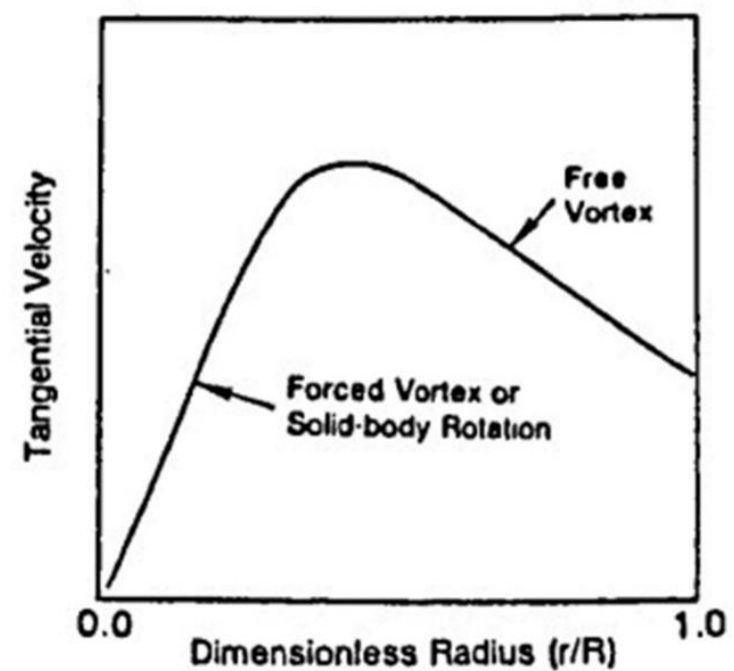

Figure 8 Qualitative representation of a Rankine vortex [27]

For this calculation of the vortex tube, the time-averaged tangential velocity, defined as $\bar{u}_{t}=\frac{1}{\tau} \int_{t=t_{0}}^{t=t_{0}+\tau} u_{t} d t$, distribution of four different section planes is shown in Figure 9. Although the absolute magnitude of the tangential velocity is different, the varying trend of the tangential velocity versus radial location is similar by comparing the plots in Figure 9. The forced vortex characteristic is shown by an almost linear relationship between the tangential velocity and radial location from the centerline to half of the radius. Then, a maximum tangential velocity is reached and then the velocity drops, demonstrating a free vortex. The flow within the vortex tube could be classified 


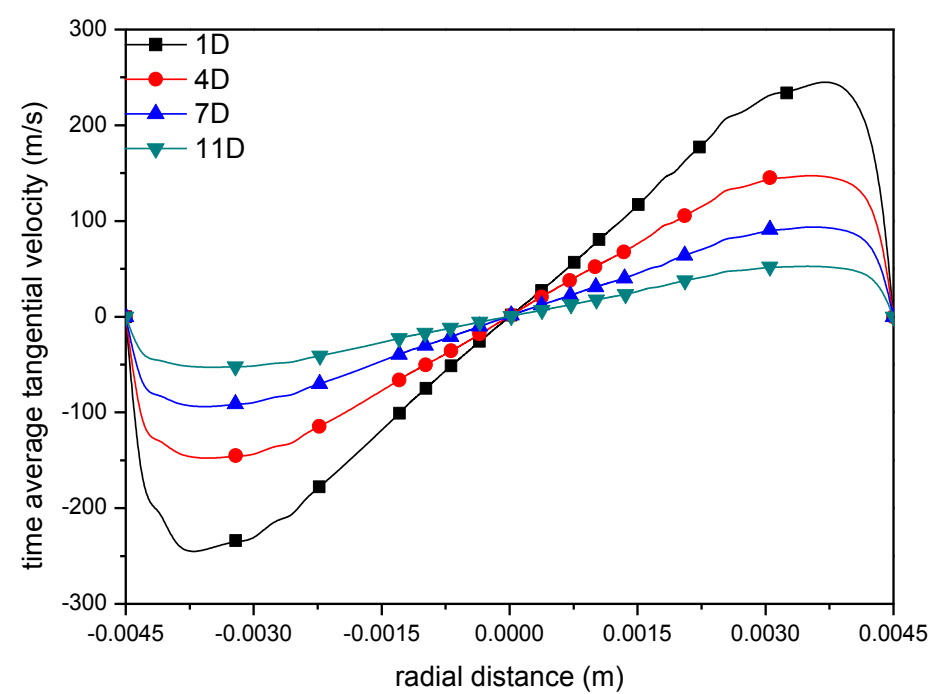

Figure 9 Radial profiles of time averaged tangential velocity at 1D, 4D, 7D and 11D (case for Pin=260kPa, $\xi=0.3$, and time average was covered for $0.05 \mathrm{~s}$ )

The swirl flow intensity can be described as the Swirl Number $S$, defined as [28],

where $r_{\text {edge }}$ is the radial distance from the axis to the edge.

$$
S=\frac{G_{\phi}}{G_{x} r_{\text {edge }}}
$$

$$
G_{\phi} \text { is the axial flux of angular momentum }=G_{\phi}=\int_{0}^{R}(w r) \rho u 2 \pi r d r
$$$$
G_{x} \text { is the axial flux of linear momentum }=G_{x}=\int_{0}^{R} u \rho u 2 \pi r d r+\int_{0}^{R} p 2 \pi r d r
$$

Based on the geometry of the inlet and the tube, a geometrical swirl number $S_{g e o}$ is derived [29], which is 3.09 for the vortex tube in this study.

$$
S_{\text {geo }}=\frac{(R-h / 2) A_{\text {tube }}}{R A_{\text {inlet }}}=\frac{(R-h / 2) \pi R^{2}}{R(4 w h)}=3.09
$$

Swirl flow was classified into weak swirl flows $(S<0.6)$ and strong swirl flows ( $S \geq 0.6$ ) according to the swirl number [25]. When the swirling flow is generated, a natural radial pressure gradient, due to the term $w^{2} / r$, is established by centrifugal effects and expansion through the nozzle causes the radial distribution of centrifugal pressure gradient to decay in the axial direction [27]. At low swirl levels, the axial pressure gradient is not strong enough to cause axial recirculation, but when increasing the swirl, strong coupling develops between axial and tangential velocity components. 
This coupling induces reverse flow and a central recirculation zone $(C R Z)$ with a stagnation point is formed on the vortex axis. The appearance of $C R Z$ is a distinct sign of vortex breakdown in swirl flows. Leibovich [30] describes vortex breakdown as an intermediate phenomenon between weak and strong swirl flow, the difference is whether reverse flow occurs. Leibovich explains that vortex breakdown is inherently nonlinear, which is why a coherent explanation of the reason for vortex breakdown is not achieved.

After vortex breakdown occurs, the $C R Z$ becomes unstable and begins to precess around the axis and induce a large scale flow structure that contains three-dimensional time dependent instability called the precessing vortex core $(P V C)$. Syred and Beer [31, 32] revealed that the $P V C$ lies on the boundary of the reverse flow zone between the zero velocity and zero streamline. The vortex breakdown and $P V C$ are usually not only asymmetric but also highly time dependent, and they may excite and/or couple with certain acoustic modes that may lead to pressure oscillations. Vortex breakdown $P V C$ as a large vortex structure can alter the fluid dynamic behavior and make the entire flow field demonstrate unsteadiness [27, 33].

\subsection{Instability of vortex breakdown in the vortex tube}

For the vortex tube in this paper, the swirl number (3.09) is much larger than the critical swirl number ( $S=0.6$ ), which indicates that the flow in the vortex tube is strong swirl flow, and the $C R Z$ exists along with the vortex breakdown and the $P V C$. Simulation of these oscillations was observed with two monitoring points of the coordinates $(0,0,0.01)$ and $(0,0.0035,0.01)$ for the case of $P_{i n}=260.0 \mathrm{kPa}$ and $\xi=0.3$, using a transient $R S M$ model. Typical periodic structures evolved in the swirl flow when steady fluctuation occurred. A section of the time series for axial velocity at the two monitoring points is shown in Figure 10. The monitoring points were located inside and outside of the $C R Z$. 


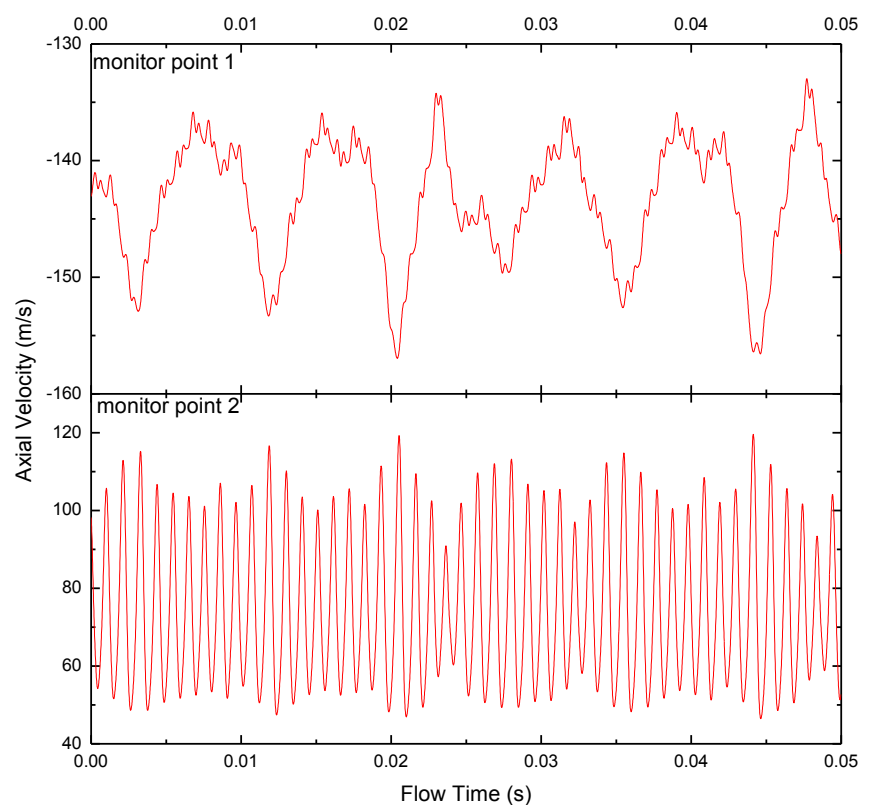

Figure 10 Time series of the monitoring points by axial velocity obtained from T-RSM

The time evolutions of both monitoring points were dominated by periodic patterns, but the oscillation modes of the points were different. To analyze the timeseries magnitude, a fast Fourier transform (FFT) [34] was used. The two monitoring points in Figure 10 show typical vibration periods at approximately $0.01 \mathrm{~s}$ and $0.001 \mathrm{~s}$. Because the unsteady computation was taken at a time step of $10^{-5} \mathrm{~s}$, the axial velocity series should contain at least 1,000 data points for input to the FFT method to analyze the frequency and power spectrum. Two series of time-continuous axial velocities were derived from FLUENT calculation results, and the FFT tools of MATLAB were adopted to carry out the analysis. As shown in Figure 11, the frequency and power spectrum density of each point differed significantly. For monitor point 1, located on the axis far from the boundary of $C R Z$, the peak frequency is $97.66 \mathrm{HZ}$ and the power spectrum density is 5.1 . For monitor point 2 , located near the boundary of $C R Z$ and significantly influenced by the $P V C$, the peak frequency is $1025.39 \mathrm{HZ}$ and the power spectrum density is 23.04. (The peak frequency at the same point is unified by monitoring any direction of velocity.) 

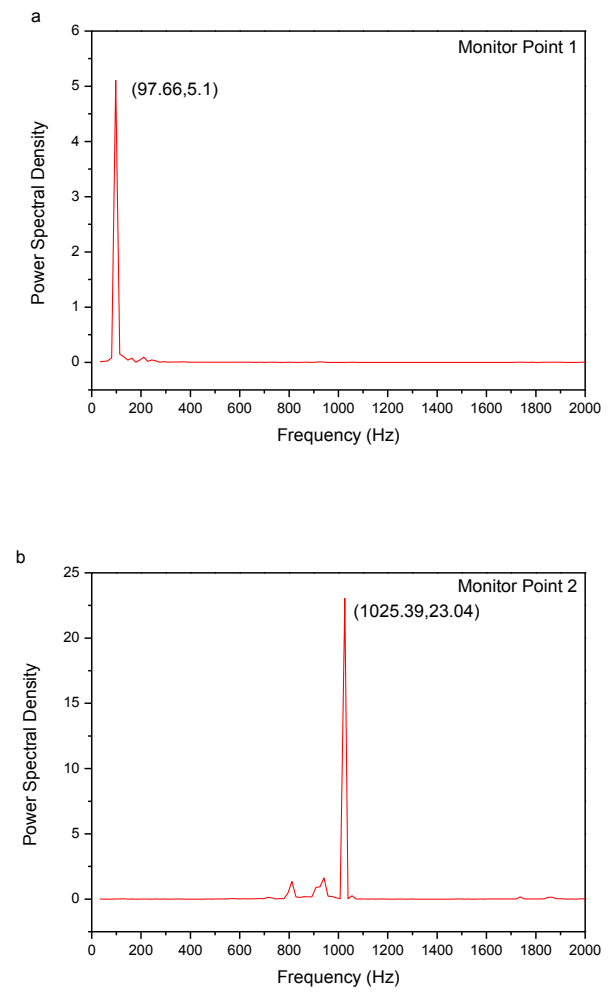

(a: monitor point 1; b: monitor point 2)

A complete period of the quasi-periodic flow structures is shown in Figure 12 by nine instantaneous contour plots of the axial (Z-direction) velocity component in an azimuthal plane $20 \mathrm{~mm}$ downstream of the inlet. Syred [33] noted that the PVC can affect the neighboring fluid flow to present precession. As shown in Figure 12, the region of reverse flow $\left(u_{z}<0\right)$ and the outer layer flow $\left(u_{z}>0\right)$ precess around the axis of symmetry. 

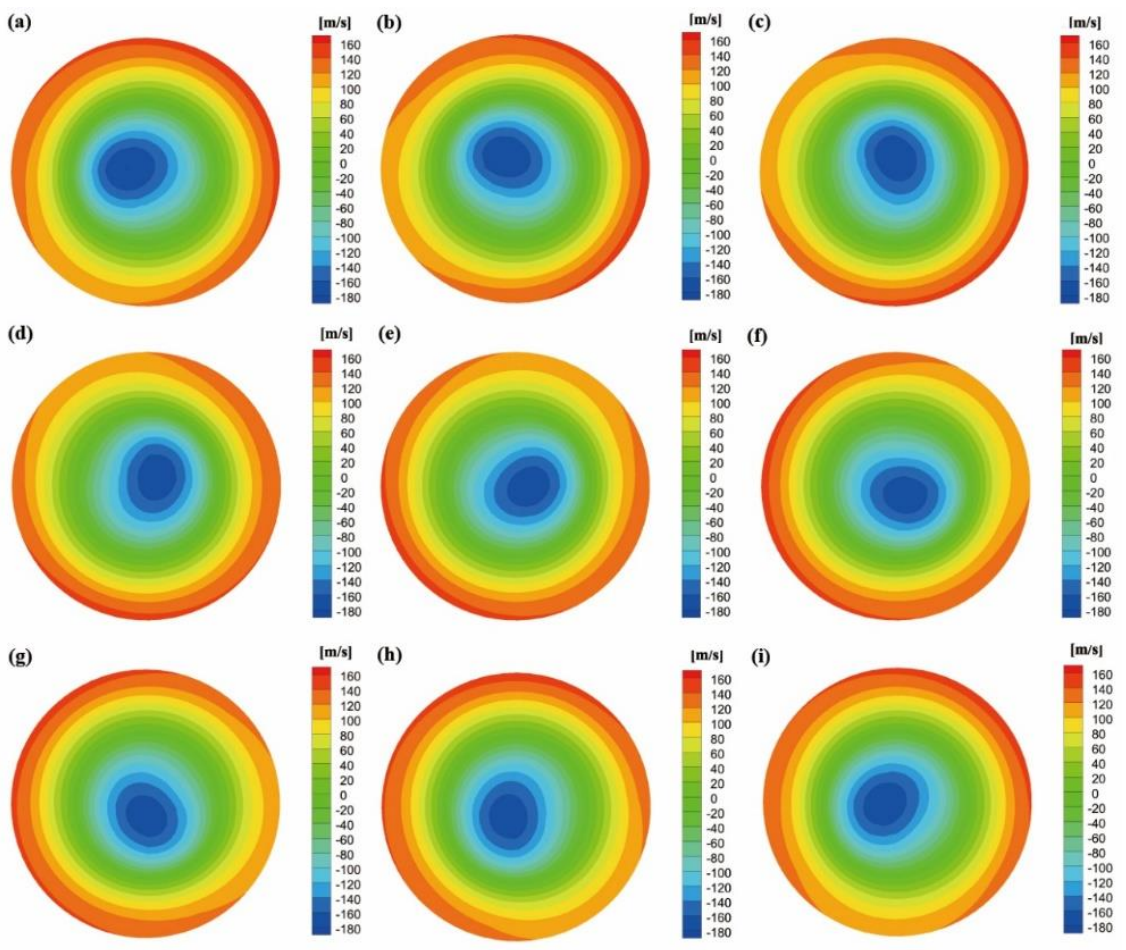

Figure $12 \mathrm{~A}$ period (top left to bottom right) of axial velocity fluctuations in the azimuthal plane $20 \mathrm{~mm}$ downstream the inlet

Figure 13 Iso-surfaces of the axial velocity

$$
\varphi=2 \pi \frac{6}{9} \text {, (h) } \varphi=2 \pi \frac{7}{9} \text {, (i) } \varphi=2 \pi \frac{8}{9} \text {. }
$$

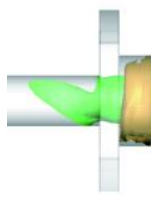

For better understanding of the flow structures, a three-dimensional structure of

the predicted flow is shown in Figure 13, where two iso-surfaces of the axial velocity are plotted. The iso-surface of the fluid emerging from the vortex tube (axial velocity $u_{z}=105 \mathrm{~m} / \mathrm{s}$ ) shown in yellow with a distinct spiral structure, extends to the hot end and damps out around the midway point of the tube. The iso-surface for $u_{z}=-25 \mathrm{~m} / \mathrm{s}$ is marked in green and flows toward the cold end. The helicity degree for the green isosurface is not as strong as the yellow iso-structure, which is nearly axisymmetric and resembles a bubble. 

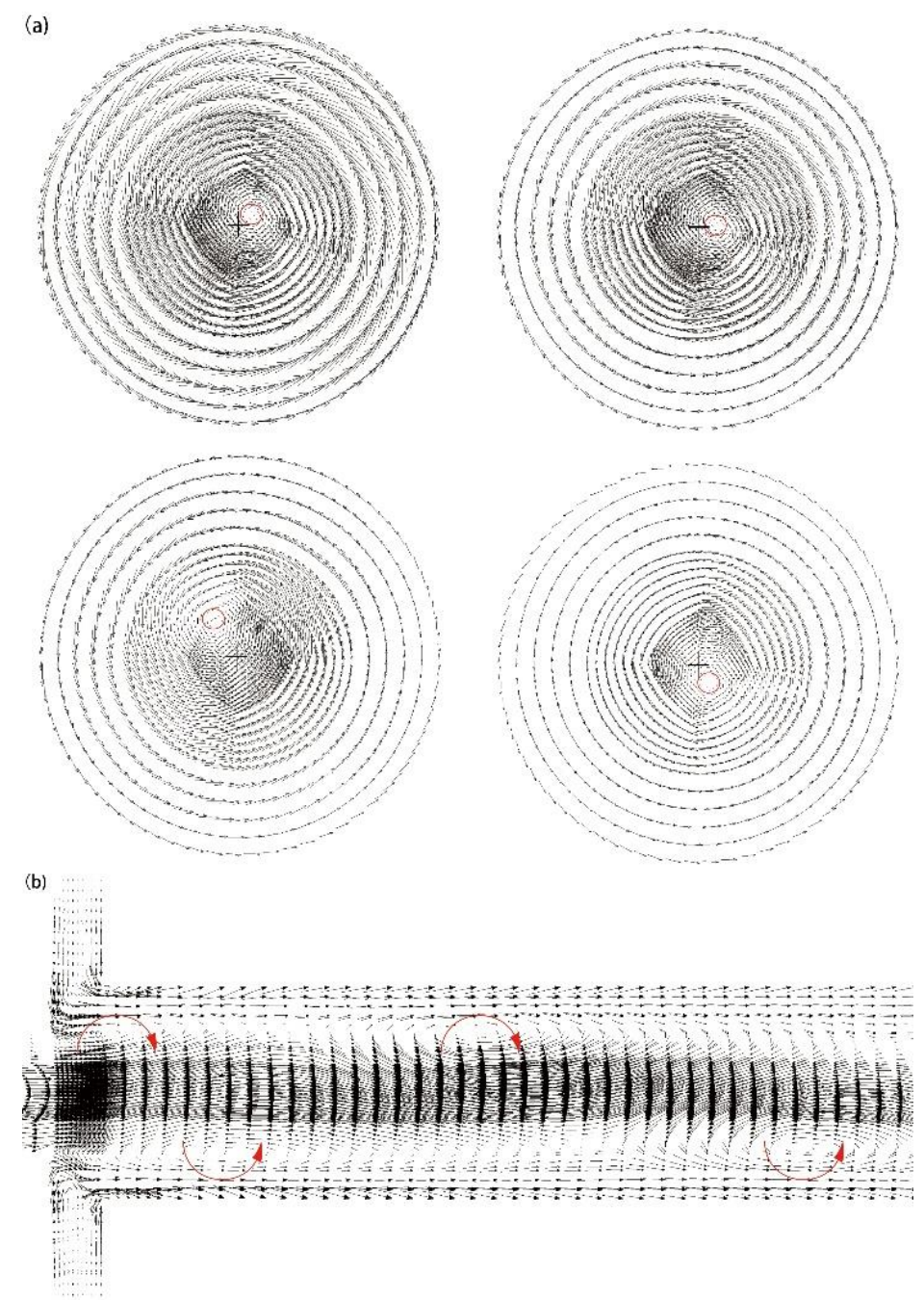

Figure 14 Flow structure oscillation patterns

(a) Sequence of snapshots showing vector plots of velocity in four planes at 1D, 4D, 7D and 11D, (b) The first half of the formation of large-scale vortex structures by vector on the meridional plane.

In Figure 14 (a), four snapshots of axis velocity vector on section planes of 1D, 4D, 7D and $11 \mathrm{D}$, are shown, from which the rotating $P V C$ process is clearly captured. The center of the vortex tube is marked by black crosses, and the vortex core is marked by red circles. The vortex cores deviated from the center of the tube and moved around the centerline. It is notable that no converged stationary solution could be obtained which also indicated the unsteady nature of the flow under consideration. In the numerical study of Kazantseva et al. [35], similar results to Figure 14(b) were found. Large-scale vortex structures were arranged periodically along the axis were considered to form the quasi-cooling cycles, and mass transfer by these vortex structures contributed to the temperature separation in the vortex tube. 


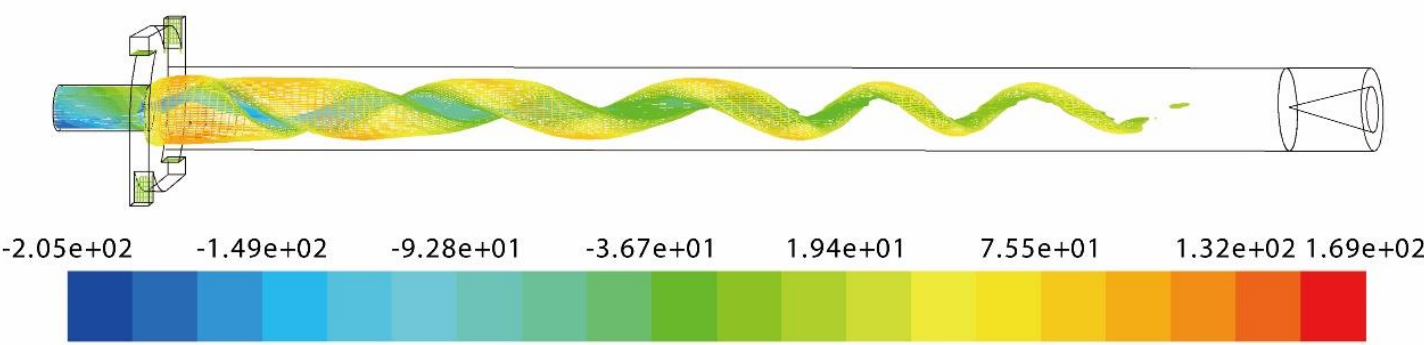

Figure 15 Iso-axial-vorticity surface colored by axial velocity.

To make identify a vortex, the iso-axial-vorticity $\left(\omega=-80000 \mathrm{~s}^{-1}\right)$ surface colored by axial velocity is shown in Figure 15. The iso-axial-vorticity surface, which is mostly concentrated on the axis of the main tube and does not emerge in the tube wall, successfully captured the high intense helicity of the large-scale vortex structure. The axial velocity contour colored the outside of the vortex structure with positive velocities and the vortex structure inside the axial velocity with negative velocities.

\section{Discussion and conclusion}

Through the above analysis based on vortex dynamics, the flow domain in the vortex tube was divided into two parts, and some pressurized stream entered the low pressure zone in the center, flowed reversely and discharged through the cold exit. Mass transfer between the cold stream and hot stream does not exist. Thus, the heat or energy transfer, according to the temperature gradient, is not feasible for explaining the temperature increase of the hot stream nor the temperature decrease of the cold stream, due to convective or conductive heat transfer on their respective streamline. 


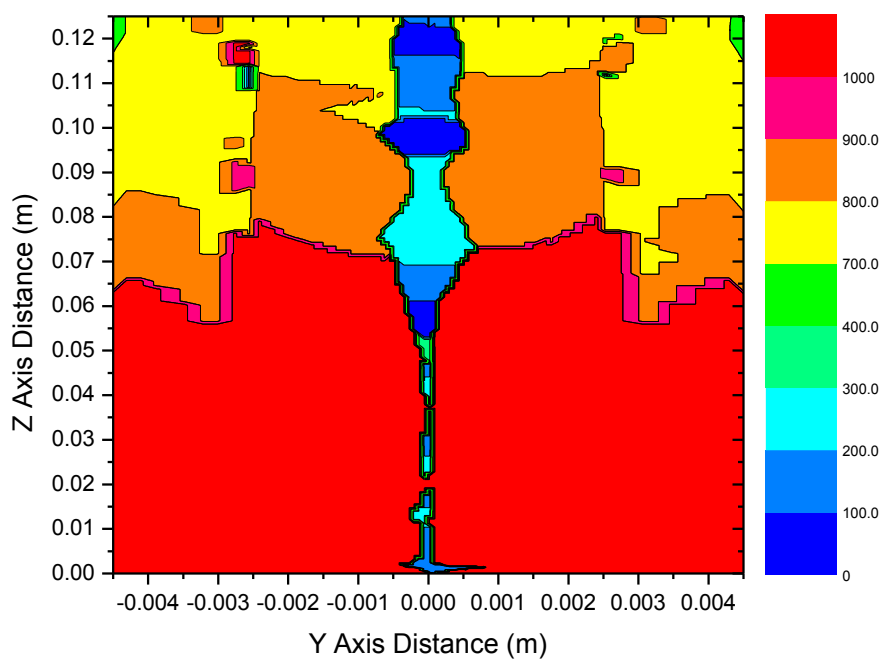

341

Figure 16 Contour of peak frequency on meridional plane in main tube (case for Pin=260kPa, $\xi=0.3$ )

The precessing vortex core in the vortex tube is always accompanied with observable phenomenon, acoustic streaming. This phenomenon has been experimentally verified to be associated with the energy separation effect [11], but no mathematical or numerical study on the $P V C$ process has been presented. In this paper, a procedure for how $P V C$ occurs and the relationship between the fluid flow and $P V C$ were provided, which revealed its mechanism numerically. The typical frequencies of 11,703 points on a section plane of the vortex tube with a fast Fourier transform method were obtained based on time-serial numerical data, and the contour of these peak frequencies are shown in Figure 16. The peak frequency ranged from $97.7 \mathrm{~Hz}$ to 1025.4 Hz. In the upstream, from the inlet to the middle of the tube, the peak frequency reached a maximum of $1025.4 \mathrm{HZ}$, which shares a proportion of $56.3 \%$. The peak frequency values decreased downstream, to demonstrate $P V C$ has a larger effect on the fluid flow near the inlet region. The lower peak frequency region is located near the centerline due to the distance between the centerline and the $C R Z$ boundary where $P V C$ is located. According to the influence intensity from $P V C$, fluid flow in the vortex tube can be divided into two regions, the heat transfer zone in the upstream and the decay of swirl zone in the downstream. A schematic of the energy transfer process is shown in Figure 17. 
Based on the results of vortex instability in the vortex tube, it can be determined that the $P V C$ dynamic contributes to energy transfer from the inner core to the highpressure, high-temperature zone. The movement, or vibration, of the boundary of the reverse flow zone is the primary mechanism for energy transfer. When the boundary layer vibrates, the particles on the layer travel between the higher pressure and lower pressure zones. Then, the particles are compressed and release heat in the high-pressure zone and are inflated and absorb heat in the low-pressure zone. The energy to keep the process stable comes from potential energy of pressurized gas as a thermal acoustic process [36]. When the oscillations are restrained, the energy transfer process will cease [11]. The energy transfer hypothesis presented is linked to thermal acoustic theory[36, 37]. Quantitative analysis and derivation of the equations for the hypothesis is future work to be completed. Energy or temperature transfer due to flow instability, and not only temperature gradient driven, may also develop as a contribution to analysis of heat and mass transfer theory.

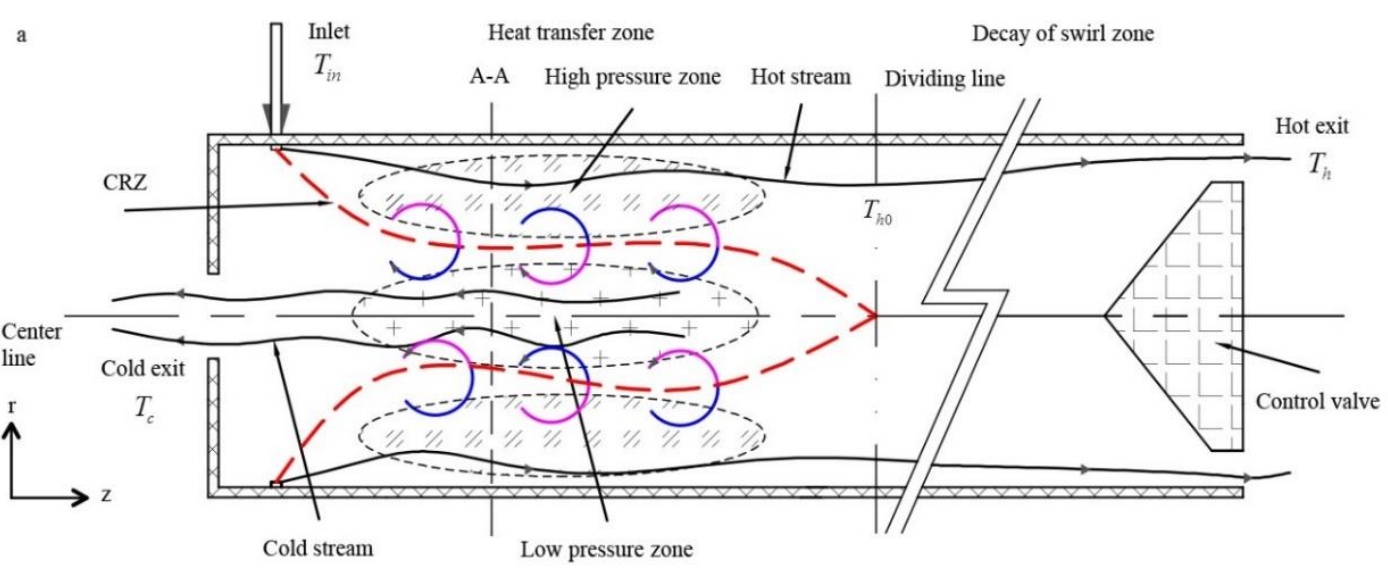

Figure 17 Schematic of the energy transfer mechanism

The unsteady analytical approach employed in this paper focuses on the periodical oscillation characteristics of the boundary when the flow field conducts limited circle oscillations. This approach differs from steady or time-averaged flow fluid studies [3840], in which the reverse flow boundary is considered stationary at a certain operating condition when the flow field achieves steady state. The particles around the boundary affected by $P V C$ move back-and-forth between the periphery and inner layers at certain frequencies and serve as the medium to transfer energy from the cold stream to the hot 
stream. However, mass transfer between layers does not necessarily exist because the integration of mass transfer on the entire boundary per period is zero in the unsteady flow field. There is no contradictory between steady and unsteady studies on this point, nevertheless, unsteady studies take the advantage to reveal the microscopic process and establish a connection between flow structure and heat transfer mechanism.

\section{ACKNOWLEDGMENTS}

Our research program in this paper was supported by the National Science Foundation of China under grant (No. 51276025).

\section{REFERENCES}

[1] R. Georges, Method and apparatus for obtaining from alpha fluid under pressure two currents of fluids at different temperatures, in, Giration, Des Fluides Sarl US, 1934.

[2] A.F. Gutsol, The Ranque effect, Physics-Uspekhi, 40(6) (1997) 639-658.

[3] S. Eiamsa-ard, P. Promvonge, Review of Ranque-Hilsch effects in vortex tubes, Renewable and Sustainable Energy Reviews, 12(7) (2008) 1822-1842.

[4] Y. Xue, M. Arjomandi, R. Kelso, A critical review of temperature separation in a vortex tube, Experimental Thermal and Fluid Science, 34(8) (2010) 1367-1374.

[5] H.R. Thakare, A. Monde, A.D. Parekh, Experimental, computational and optimization studies of temperature separation and flow physics of vortex tube: A review, Renewable and Sustainable Energy Reviews, 52 (2015) 1043-1071.

[6] B.K. Ahlborn, J.M. Gordon, The vortex tube as a classic thermodynamic refrigeration cycle, Journal of Applied Physics, 88(6) (2000) 3645-3653.

[7] J.U.K. B. K. Ahlborn, E. Rebhan, The heat pump in a vortex tube, J. Non-Equilib. Thermodyn., 23 (1998) 159-165.

[8] Y. Xue, M. Arjomandi, R. Kelso, Experimental study of the flow structure in a counter flow Ranque-Hilsch vortex tube, International Journal of Heat and Mass Transfer, 55(21-22) (2012) 5853-5860.

[9] Y. Xue, M. Arjomandi, R. Kelso, The working principle of a vortex tube, International Journal of Refrigeration, 36(6) (2013) 1730-1740.

[10] Y. Xue, M. Arjomandi, R. Kelso, Visualization of the flow structure in a vortex tube, Experimental Thermal and Fluid Science, 35(8) (2011) 1514-1521.

[11] M. KUROSAKA, Acoustic streaming in swirling flow and the Ranque-Hilsch (vortex-tube) effect Journal of Fluid Mechanics, 124 (1982) 139-172.

[12] R. Liew, J.C. Zeegers, J.G. Kuerten, W.R. Michalek, Maxwell's demon in the Ranque-Hilsch vortex tube, Phys Rev Lett, 109(5) (2012) 054503.

[13] R. Liew, Droplet Behaviour and Thermal Separation in Ranque-Hilsch Vortex Tubes Technische Universiteit Eindhoven, 2013.

[14] S.B. K. Dincer, B.Z. Uysalc, I. Ucguld, Experimental investigation of the performance of a Ranque-Hilsch vortex tube with regard to a plug located at the hot outlet, International Journal of 
Refrigeration, 32 (2009) 87-94.

[15] M.B. A. Ouadha , Y. Addad Effects of variable thermophysical properties on flow and energy separation in a vortex tube, International Journal of Refrigeration, 36 (2013) 2426-2437.

[16] A.O. Mohammed Baghdad, Omar Imine, Yacine Addad Numerical study of energy separation in a vortex tube with different RANS models, International Journal of Thermal Sciences, 50(12) (2011) 2377-2385.

[17] A.M. B. Wegner , C. Schneider, A. Sadiki, A. Dreizler and J. Janicka, Assessment of unsteady RANS in predicting swirl flow instability based on LES and experiments, International Journal of Heat and Fluid Flow 25 (2004) 528-536.

[18] A.S. P. Jochmann, M. Hehle, O. Scha "fer, R. Koch, H.-J. Bauer, Numerical simulation of a precessing vortex breakdown, International Journal of Heat and Fluid Flow, 27 (2006) 192-203.

[19] P.A. Durbin, A perspective on recent developments in RANS modeling, Engineering Turbulence Modelling and Experiments, 5 (2002) 3-16.

[20] G. laccarino, A. Ooi, P.A. Durbin, M. Behnia, Reynolds averaged simulation of unsteady separated flow, International Journal of Heat and Fluid Flow, 24(2) (2003) 147-156.

[21] B.J. Daly, F.H. Harlow, Transport equations in turbulence, Physics of Fluids (1958-1988), 13(11) (1970) 2634-2649.

[22] B.E. Launder, Second-moment closure and its use in modelling turbulent industrial flows, International Journal for Numerical Methods in Fluids, 9(8) (1989) 963-985.

[23] B.E. Launder, D. Spalding, The numerical computation of turbulent flows, Computer methods in applied mechanics and engineering, 3(2) (1974) 269-289.

[24] B.F.a.A.G. T. Farouk, Simulation of gas species and temperature separation in the counterflow Ranque-Hilsch vortex tube using the large eddy simulation technique, International Journal of Heat and Mass Transfer, 52(13-14) (2009) 3320-3333.

[25] A.K. Gupta, D.G. Lilley, N. Syred, Swirl flows, Abacus Press, 1984.

[26] O. Lucca-Negro, Modelling of swirl flow instabilities, University of Wales Cardiff, 1999.

[27] T.O.D. O. Lucca-Negro, Vortex breakdown a review, Progress in Energy and Combustion Science, 27 (2001) 431-481.

[28] D.G. LILLEY, Prediction of Inert Turbulent Swirl Flows, AIAA Journal, 11(7) (1973) 955-960.

[29] C. Biegger, C. Sotgiu, B. Weigand, Numerical investigation of flow and heat transfer in a swirl tube, International Journal of Thermal Sciences, 96 (2015) 319-330.

[30] S. Leibovich, The Structure of Vortex Breakdown, Ann. Rev. Fluid Mech, 10 (1978) 221-246.

[31] N. Syred, J. Beer, Combustion in swirling flows: a review, Combustion and flame, 23(2) (1974) 143-201.

[32] N. Syred, J. Beer, Damping of precessing vortex cores by combustion in swirl generators, Astronautica Acta, 17(4-5) (1972) 783.

[33] N. Syred, A review of oscillation mechanisms and the role of the precessing vortex core (PVC) in swirl combustion systems, Progress in Energy and Combustion Science 32 (2006) 93-161.

[34] J.W.C.a.J.W. Tukey, An algorithm for the machine calculation of complex Fourier series, Mathematics of computation, 19(90) (1965) 297-301.

[35] S.A.P. O. V. Kazantseva, and A. A. Fuzeeva, Numerical Simulation of Swirling Flows in Vortex Tubes, High Temperature, 43 (2005) 606-611.

[36] G.W. Swif, Thermoacousticengines, J. Acoust.Soc. Am., 84(4) (1988) 1145-1180.

[37] M.E.H. Tijani, Loudspeaker-driven thermoacoustic refrigeration, Technische Universiteit 
Eindhoven, 2001.

[38] G.F.N.a.S.A.K. N. F. Aljuwayhel, Parametric and internal study of the vortex tube using a CFD model, International Journal of Refrigeration, 28(3) (2005) 442-450.

[39] M.A.a.R.K. Yunpeng Xue, Experimental study of the flow structure in a counter flow RanqueHilsch vortex tube, International Journal of Heat and Mass Transfer, 55 (2012) 5853-5860.

[40] M.A.a.R.K. Yunpeng Xue, The working principle of a vortex tube, International Journal of Refrigeration, 36(6) (2013) 1730-1740.

\section{FIGURES LIST}

Figure 1 Geometry model: a. schematic representation and dimensions (in $\mathrm{mm}$ ); b. axis system from Baghdad et al.

Figure 2 Computational mesh of the vortex tube.

Figure 3 Performance of T-RSM in predicting experimental measurements and comparing with RSM.

Figure 4 Grid size dependence study on time average total temperature separation....10

Figure 5 Instantaneous axial velocity contours in the meridional plane.

Figure 6 Time averaged axial velocity contours in the meridional plane

Figure 7 Radial profiles of time averaged axial velocity at 1D, 4D, 7D and 11D .............. 11

Figure 8 Qualitative representation of a Rankine vortex [27]

Figure 9 Radial profiles of time averaged tangential velocity at 1D, 4D, 7D and 11D ....13

Figure 10 Time series of the monitoring points by axial velocity obtained from T-RSM15

Figure 11 Power spectral densities and frequencies obtained by FFT.

Figure $12 \mathrm{~A}$ period (top left to bottom right) of axial velocity fluctuations in the azimuthal

plane $20 \mathrm{~mm}$ downstream the inlet.

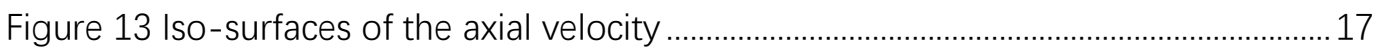

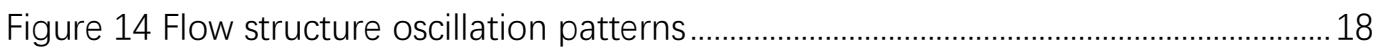

Figure 15 Iso-axial-vorticity surface colored by axial velocity ............................................. 19

Figure 16 Contour of peak frequency on meridional plane in main tube ..........................20

Figure 17 Schematic of the energy transfer mechanism .......................................................2 21 